\title{
Potential Antimigraine Effects of Warfarin: An Exploration of Biological Mechanism with Survey of Patients
}

\author{
Benjamin Nilsson ${ }^{1,2}$ Valentina Back ${ }^{2,3}$ Ran Wei ${ }^{3,4}$ \\ Tammy J. Bungard ${ }^{6, *}$ \\ ${ }^{1}$ Alberta Health Services, Edmonton, Alberta, Canada \\ 2 Faculty of Pharmacy and Pharmaceutical Sciences, University of \\ Alberta, Edmonton, Alberta, Canada \\ ${ }^{3}$ Cardiovascular Research Centre, University of Alberta, Edmonton, \\ Alberta, Canada \\ ${ }^{4}$ Department of Pharmacology, Faculty of Medicine and Dentistry, \\ University of Alberta, Edmonton, Alberta, Canada \\ ${ }^{5}$ Alberta Mazankowski Heart Institute, University of Alberta, \\ Edmonton, Alberta, Canada \\ ${ }^{6}$ Department of Cardiology, Faculty of Medicine and Dentistry, \\ University of Alberta, Edmonton, Alberta, Canada
}

\author{
Address for correspondence Paul Jurasz, PhD, Faculty of Pharmacy \\ and Pharmaceutical Sciences, 3-142E Katz Group-Rexall Centre, \\ 11361-87 Avenue, Edmonton, AB, Canada T6G 2E1 \\ (e-mail: jurasz@ualberta.ca).
}

TH Open 2019;3:e180-e189.

\begin{abstract}
Case reports suggest a link between anticoagulant use and improved migraine symptoms, and a role for platelet-induced cerebral vasoconstriction in migraine pathobiology. Hence, we investigated the mechanism by which warfarin may affect migraine symptoms and whether there is a change in migraine symptomology in patients initiating oral anticoagulants, most commonly warfarin. The effects of warfarin on human platelet aggregation and secretion as well as platelet-induced rat cerebral artery vasoconstriction were studied. A survey of migraine and symptom change after starting or stopping oral anticoagulants was also conducted. Warfarin inhibited platelet aggregation and 5-hydroxytryptamine (5-HT) secretion in a concentration-dependent manner. Warfarin-inhibited platelet secretion products constricted middle cerebral arteries from male but not from female rats. For the survey, patient demographic information, migraine and medical history, and Migraine Disability Assessment Score (MIDAS) changes were collected. Out of 175 consenting, 40 respondents met the criteria for migraine and completed the survey. A total of 11 patients reported migraine symptom change, all coinciding with starting warfarin. Of those having symptom and MIDAS improvement, most were female with migraines

Keywords

- warfarin

- migraine

- platelets

- serotonin

- blood vessels with aura, whereas those worsening were male with fewer having migraine with aura. Of those reporting migraine symptom change with warfarin, female sex may be associated with improved MIDAS, and those experiencing an aura component are more likely to report a symptom change. Warfarin-mediated symptom improvement in females may occur due to inhibition of platelet $5-\mathrm{HT}$ secretion and a lower sensitivity of female cerebral blood vessels to platelet-derived 5-HT-induced vasoconstriction.
\end{abstract}

\footnotetext{
Co-senior authors.
}

received

April 1, 2019

accepted after revision

May 20, 2019
DOI https://doi.org/

$10.1055 / \mathrm{s}-0039-1692989$.

ISSN 2512-9465. (c) 2019 Georg Thieme Verlag KG

Stuttgart · New York
License terms

(c) (i) 


\section{Introduction}

The pathogenesis of migraine is unclear. Based on early reports, it was proposed that the aura with migraines (classical migraine) was caused by cerebral vasoconstriction that then proceeded to a reactive intracranial and extracranial vasodilation with the associated headache. ${ }^{1}$ Investigations of cerebral blood flow have not fully supported this early theory, as transition from vasoconstriction to vasodilatory hyperemia does not necessarily coincide with the onset of headache and as the hyperemia may persist following headache disappearance. ${ }^{2,3}$ Subsequently, it has been hypothesized that migraines occur due to neurovascular mechanisms that lead to dysfunction in neuronal and broad sensory processing due to activation of the trigeminovascular system and neurogenic inflammation. ${ }^{4}$ In addition to the reported cerebral hemodynamic changes associated with migraine, it has been suggested that platelets may also be involved in migraine pathogenesis. ${ }^{5}$ Several studies have demonstrated enhanced platelet aggregation and 5-hydroxytryptamine (5-HT) secretion in migraineurs. ${ }^{6-9} 5-\mathrm{HT}$ is thought to play an important role in migraine pathogenesis, as it is capable of modulating both pain transmission and vascular tone. ${ }^{10}$

Previous studies have also shown that antiplatelet medications can have a demonstrable effect on migraine symptoms. One chart review of hospitalized patients found that acetylsalicylic acid (ASA) reduces the number of migraine with aura episodes by up to six-fold, ${ }^{11}$ while another showed that clopidogrel reduces migraine with aura following closure of persistent foramen ovale and atrial septal defects. ${ }^{12}$ Similarly, several case reports have also associated an improvement in migraine symptomatology with the use of vitamin $\mathrm{K}$ antagonists (VKAs). ${ }^{13-17}$ We recently reported a case wherein remission of migraines was maintained throughout 12 years of warfarin therapy, recurred when the female patient was switched to apixaban, and then again resolved with the reintroduction of warfarin. ${ }^{18}$ As some anticoagulants have been shown to also have antiplatelet effects, ${ }^{19-21}$ platelets and their ability to secrete 5-HT are an attractive potential mechanism linking the activity of anticoagulant medications to potential changes in migraine symptoms. In support of such a mechanistic link, we sought to investigate the effect of warfarin on platelet function, 5-HT release, and vascular tone to determine whether there is a biologically plausible mechanism linking anticoagulant use to relief of migraine symptoms, as well as to survey patients to determine whether any patients noticed a change in migraine symptomatology with alteration in oral anticoagulant therapy within an anticoagulation clinic (AC). To our knowledge, the assessment of change in migraine symptoms has not been assessed in a larger population taking VKAs, even though an association between migraine with aura and an increased risk of ischemic stroke has been identified, particularly in females. ${ }^{22,23}$

\section{Methods}

\section{Platelet Isolation and Aggregation}

Approval for the study was obtained from the University of Alberta Research Ethics Board. Following informed consent, blood was collected from healthy volunteers who had not taken any drugs affecting platelet function for 14 days prior to the study. Prostacyclin (prostaglandin $\mathrm{I}\left[\mathrm{PGI}_{2}\right]$ )-washed platelets $\left(2.5 \times 10^{8} / \mathrm{mL}\right)$ were prepared in Tyrode's buffer, and platelet aggregation in response to collagen $(0.6 \mu \mathrm{g} / \mathrm{mL})$ was measured as light transmittance percentage in a lumiaggregometer (Chronolog, Havertown, Pennsylvania, United States) as described previously. ${ }^{24}$ The inhibitory effects of warfarin $(0-30 \mu \mathrm{g} / \mathrm{mL})$ on platelet aggregation were normalized and extent of aggregation expressed as percent of vehicle (saline) control. After aggregation, platelet releasates were separated from pellets by adding $1 \mu \mathrm{g} / \mathrm{mL} \mathrm{PGI}_{2}$ to aggregated samples, followed by centrifugation $(10,000 \mathrm{~g}$ for 5 minutes). The releasates were then stored at $-80^{\circ} \mathrm{C}$ prior to further analysis.

\section{Enzyme-Linked Immunosorbent Assay}

As markers of platelet $\alpha$ - and $\delta$-granule secretion, vascular endothelial cell growth factor $\left(\mathrm{VEGF}_{165}\right)$ and serotonin (5-HT) were quantified in platelet releasates using the Quantikine ELISA Kit for human VEGF (R\&D Systems, Minneapolis, Minnesota, United States) and a Serotonin enzyme-linked immunosorbent assay (ELISA) kit (Enzo Life Sciences, Brockville, Ontario, Canada), respectively. ELISAs were performed according to manufacturer's instructions and absorbance measured using an iMark 96-well plate reader (Bio-Rad, Mississauga, Ontario, Canada). The effects of warfarin on platelet secretion of VEGF and 5-HT were then expressed as percent of vehicle (saline) control.

\section{Animal Care and Use}

All animal care and experimental procedures were approved by the Animal Care and Use Committee at the University of Alberta and performed in accordance with Canadian Council on Animal Care guidelines. Male Sprague-Dawley rats (250-300 g; Bioscience, University of Alberta) were housed in an enriched environment maintained on a 12:12-hour light-dark cycle at approximately $23^{\circ} \mathrm{C}$ with fresh tap water and standard chow available ad libitum. Rats were euthanized by inhalation of isoflurane followed by decapitation. The middle cerebral arteries were removed postmortem and placed in cold Kreb's buffer containing $(\mathrm{mM}) \mathrm{NaCl} 119.0, \mathrm{NaHCO}_{3} 25.0, \mathrm{KCl}$ 4.7, $\mathrm{MgSO}_{4} 1.2, \mathrm{KH}_{2} \mathrm{PO}_{4} 1.18$, glucose 11 , and $\mathrm{CaCl}_{2} 2.5$.

\section{Pressure Myography}

Leak-free segments of middle cerebral artery (MCA; 2-3 mm in length) were mounted between two glass cannulae in an arteriograph chamber (Living Systems Instrumentation, Burlington, Vermont, United States) under conditions of no luminal flow. Vessels were bathed in Kreb's buffer at $37^{\circ} \mathrm{C}$ gassed with $4.92 \% \mathrm{CO}_{2}, 20.92 \% \mathrm{O}_{2}$, and balance $\mathrm{N}_{2}(\mathrm{pH} 7.4$ ) and intravascular pressure was maintained via a pressure servo-control system (PS200, Living Systems Instrumentation). Arteries were viewed through a Nikon TMS inverted microscope, and measurements of the internal diameter were made via an automated video dimension analyzer (VDA10, Living Systems Instrumentation). The glass cannulae (borosilicate glass with outer diameter of $1.2 \mathrm{~mm}$ and 
inner diameter of $0.69 \mathrm{~mm}$ ) were pulled using a Model P87 Flaming/Brown micropipette puller (Sutter Instruments, Novato, California, United States). Pressure and diameter measurements were recorded via Powerlab data acquisition system using the data acquisition software LabChart 5 (AD Instruments, Colorado, United States). MCAs which did not develop myogenic tone during an initial equilibration period of 45 minutes at $80 \mathrm{~mm} \mathrm{Hg}$ and $37^{\circ} \mathrm{C}$ were discarded. Following the equilibration period, the intravascular pressure was set to $40 \mathrm{~mm} \mathrm{Hg}$ and held at that pressure for 15 minutes before the addition of human platelet releasate into the bath. Platelet releasate was in the bath for a minimum of 15 minutes. In some experiments, ketanserin $(1 \mu \mathrm{M})$ was added to the bath 15 minutes prior to the addition of the platelet releasate. In separate experiments, arterial responsiveness to 5-HT was assessed by cumulative additions of 5 -HT ( $1 \mathrm{nM}$ to $3 \mu \mathrm{M}$ ) into the bath. The effect of releasates and 5-HT on arterial diameter was measured as the change in baseline in microns $(\mu \mathrm{m})$.

\section{Survey}

\section{Population and Survey Conduction}

Survey ethics approval was received through the Research Ethics Board at the University of Alberta. Eligible patients had their warfarin managed by the AC at the University of Alberta Hospital, Edmonton, Alberta, Canada, that manages approximately 750 patients. Participants had to be older than 18 years, have initiated the anticoagulant within 10 years, have administered the anticoagulant for the past 3 months, and have access to the Internet and be able to speak/read English. Over the course of 4 weeks, the AC staff screened and approached all contacted patients using a standardized script to participate in the survey (regardless of an awareness of migraine history) and collected email addresses for those agreeing. Four weeks was deemed an appropriate time interval given the $\mathrm{AC}$ recalls patients (at the longest) on a monthly basis. The survey was constructed using the Qualtrics software platform. Links were sent out to agreeable patients with reminders occurring at 2 and 4 weeks and the survey remained open for a total of 5 weeks. Implied consent was obtained with completion of the survey.

\section{The Survey}

The survey was divided into four sections: demographics, migraine history, anticoagulant use, and changes in migraine symptomatology associated with anticoagulant use. To have a history of migraines (and proceed with the survey), participants had to either identify an established medical diagnosis or at least 1 symptom among a list of 19 symptoms included based on the International Headache Society (IHS) Classification ICHD-11 criteria for migraine headache. ${ }^{25}$ Anticoagulant usage, including start and stop times, was identified. Finally, alteration in migraine symptomatology in relation to anticoagulant use was determined; if a change in symptoms was apparent, further information was collected using the Migraine Disability Assessment Score (MIDAS). ${ }^{25}$ The MIDAS assesses the impact migraines have on the daily level of pain and disability and contains the following: Grade 1 (score 0-5), little or no disability; Grade 2 (score 6-10), mild disability; Grade 3 (score 11-20), moderate disability; and Grade 4 (score $21+$ ), severe disability. A reduction in MIDAS over time demonstrates improvement, whereas an increase demonstrates worsening of migraine symptoms. In qualifying changes in migraine symptomatology via the MIDAS, participants were asked to focus on the 3 months prior and 3 months after the anticoagulant change. Both prophylactic and acute and migraine therapies were also collected.

\section{Survey Data Analysis}

Among those having migraines, data were separated based on whether symptom change did or did not occur with alteration in oral anticoagulant. For those reporting symptom change, further breakdown was performed based on the change to the MIDAS (improved, worsened, or not changed) and the data were described.

\section{Statistics}

Statistics were performed using GraphPad Prism 7.0 software. All means are reported with standard error of the mean. One-way analysis of variance (ANOVA) with Dunnett's post hoc test and two-way ANOVA were performed where appropriate. A $p$-value $<0.05$ was considered significant.

\section{Results}

We investigated the effects of warfarin on platelet aggregation and secretion as a possible mechanistic link between anticoagulant use and change in migraine symptoms. Warfarin inhibited platelet aggregation in response to collagen $(0.6 \mu \mathrm{g} / \mathrm{mL})$ in a concentration-dependent manner (-Fig. 1A); and at $30 \mu \mathrm{g} / \mathrm{mL}$ it caused a small but significant inhibition in aggregation $(84.3 \pm 5.5 \%$ vs. $100 \%$ control aggregation; $p<0.05$; - Fig. 1B). Interestingly, at $30 \mu \mathrm{g} / \mathrm{mL}$ warfarin had a large inhibitory effect on platelet 5-HT secretion in response to collagen $(53.0 \pm 8.4 \%$ vs. $100 \%$ control 5-HT secretion; $p<0.05$ ). An uncoupling of 5-HT secretion inhibition from inhibition of platelet aggregation by warfarin was evident from the $\mathrm{IC}_{50}$ of the two events (32.9 vs. $69.0 \mu \mathrm{g} / \mathrm{mL}$; - Fig. 1C). This type of uncoupling was not evident between inhibition of platelet aggregation and platelet VEGF secretion, as only $100 \mu \mathrm{g} / \mathrm{mL}$ warfarin significantly inhibited VEGF secretion (-Fig. 1D).

Previous case reports suggest that a sex difference may exist in migraine symptomology change with VKA therapy $^{13-18}$; however, no significant difference was observed in the effects of warfarin on 5-HT secretion from female versus male platelets. Therefore, to investigate potential differential effects of warfarin-inhibited platelet secretion on cerebral vascular tone in males versus females, pressure myography was performed on MCAs excised from male and female rats and treated with warfarin $(30 \mu \mathrm{g} / \mathrm{mL})$-inhibited platelet releasates. Warfarin-inhibited platelet releasates caused a significantly greater constriction of male versus female MCAs $(-16.99 \pm 5.57 \mu \mathrm{m} \quad$ vs. $\quad-2.30 \pm 2.41 \mu \mathrm{m}$ 

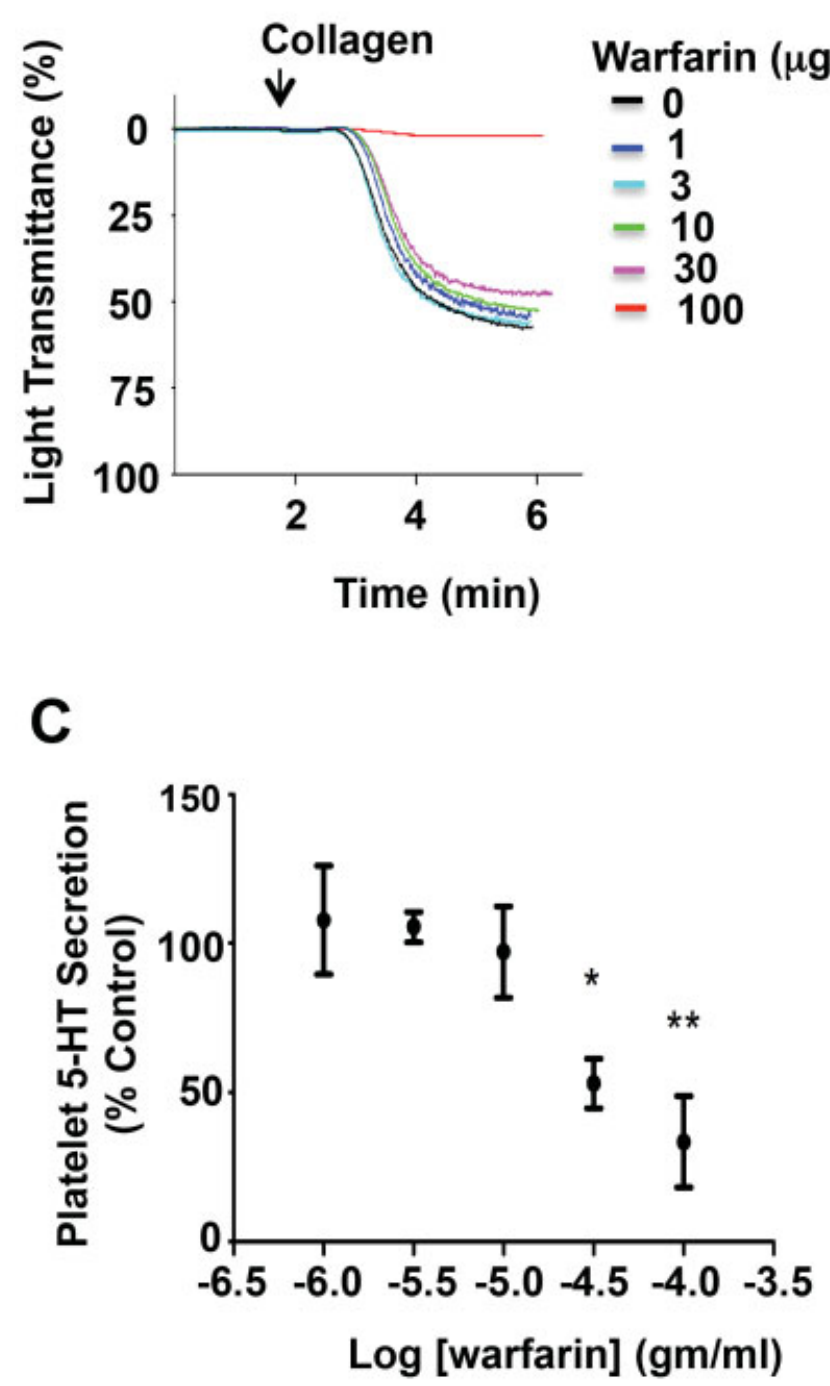

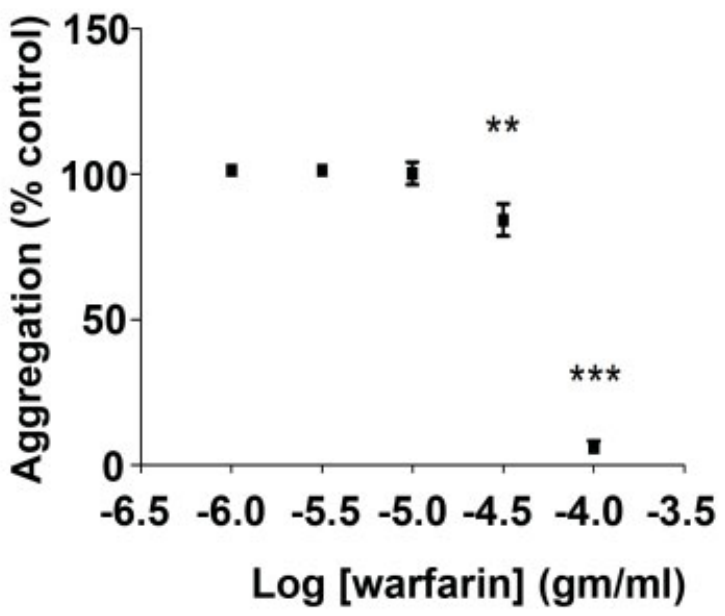

D

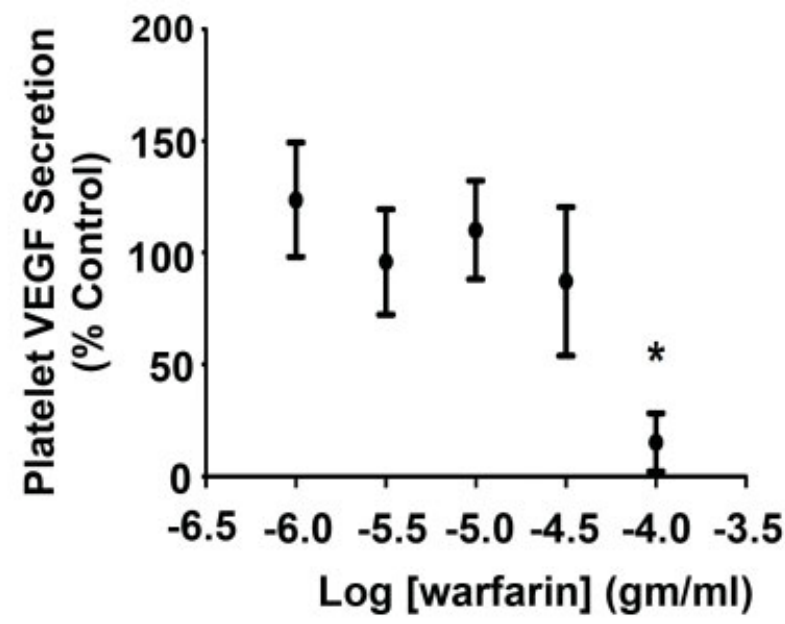

Fig. 1 (A) Representative platelet aggregometry traces and (B) summary data demonstrating the concentration-dependent inhibitory effects of warfarin on platelet aggregation. $N=5$ ( 3 female donors and 2 male donors). ${ }^{* *} p<0.01 ;{ }^{* * *} p<0.001$. (C, D) Summary data demonstrating the inhibitory effects of warfarin on platelet $5-\mathrm{HT}$ and VEGF secretion, respectively. $N=5$ ( 3 female donors and 2 male donors). ${ }^{*} p<0.05$; ${ }^{* *} \mathrm{p}<0.01$. VEGF, vascular endothelial growth factor; 5-HT, 5-hydroxytryptamine.

change in internal diameter; $p<0.05$; - Fig. 2A). This constriction of male MCAs by warfarin-inhibited platelet releasates occurred in response to platelet-secreted 5-HT as the $5-\mathrm{HT}_{2 / 1}$ receptor antagonist Ketanserin inhibited the constriction of male MCAs in response to platelet releasates $(-16.99 \pm 5.57 \mu \mathrm{m}$ vs. $-1.82 \pm 1.96 \mu \mathrm{m}$ change in internal diameter; $p<0.05$; - Fig. 2B). Consistent with this finding, concentration response experiments demonstrated that MCAs from male rats are more sensitive to the vasoconstrictive effects of $5-\mathrm{HT}$ than those from females $\left(\mathrm{EC}_{50}\right.$ of $42.1 \mathrm{vs}$. 202.7 nM; - Fig. 2C).

To determine whether our experimental findings could potentially explain changes in migraine symptomology with VKA therapy in the clinic, we screened 680 patients for inclusion into our survey of migraine and symptom change after starting or stopping oral anticoagulants. A total of 175 consented to receive the survey, with 40 patients included (-Fig. 3). Of the 40 with migraines, 11 (27.5\%) reported a change in migraine severity, frequency or duration, and all reported this change upon starting warfarin therapy. Compared with those not reporting a change in migraine status, those with a change tended to be younger (with 41.3 and $72.7 \%$ being $\leq 55$ years, respectively) and have migraine with aura (20.7 vs. $72.7 \%$; - Table 1). Both those that did not and did report a change in migraine status had several years since the onset of migraines (32 and 21 years), most commonly had warfarin indicated due to a mechanical valve (72.4 and $90.9 \%$ ), and were taking warfarin for a median of 5 and 3 years, respectively.

Among the 11 reporting a change in their migraines, assessment of this change with the MIDAS identified an improvement for 5 , worsening for 4 , and no change for the 


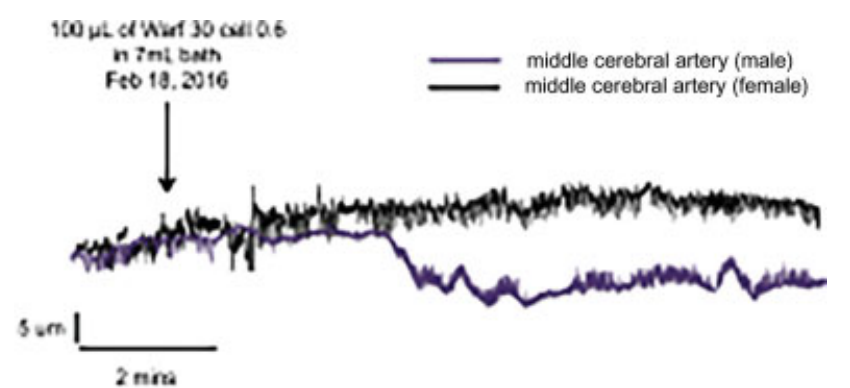

A

B
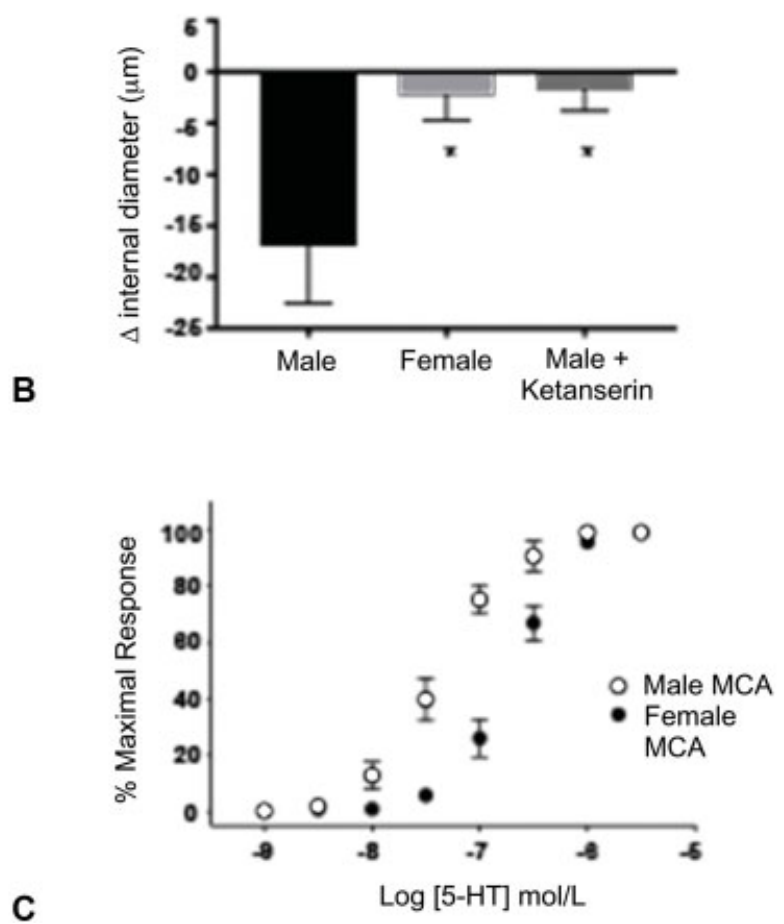

Fig. 2 (A) Representative pressure myography traces and (B) summary data demonstrating the 5-HT-dependent constrictive effects of warfarin $(30 \mu \mathrm{g} / \mathrm{mL})$ inhibited platelet releasates on male, but not on female, rat middle cerebral arteries. $N=4$ for each sex. ${ }^{*} p<0.05$. (C) Concentration responses experiments demonstrating vasoconstriction to $5-\mathrm{HT}$ of male versus female rat MCAs. $N=5$ for each sex. 5-HT, 5-hydroxytryptamine.

remaining 2 (-Table 2 ). The five with both symptom report and MIDAS change reflecting improvement had substantial changes in the MIDAS $(-19[-24,-8])$, with four reporting stopping acute migraine treatments. Notably, the majority with improvement were female $(4 / 5)$, had migraine with aura (4/5), and had a history of smoking (3/5). Those having worsening of symptoms and MIDAS change reflecting this $(N=4)$ also had substantial changes in the MIDAS (24 (14, $40)$ ), with one patient reporting initiation of an acute migraine treatment. In contrast to those with improvements, those with symptom worsening were all male (4/4), with $50 \%$ having migraine with aura.

\section{Discussion}

Whether a potential link exists between the anticoagulant effect of warfarin and resolution of migraines is unknown.
While there are two cases reporting improvement in symptoms without therapeutic international normalized ratios (INRs; one within 2-3 days of warfarin initiation ${ }^{18}$ and one while on a later warfarin dose reduced by $50 \%$ with INRs of $1.0-1.2),{ }^{13}$ a randomized open crossover study of acenocoumarol or propranolol identified only $1 / 12$ to respond to lowintensity VKA (INR: $1.5-2.0$ ). ${ }^{26}$ Moreover, a patient changing from warfarin to a direct factor $\mathrm{X}$ inhibitor (apixaban) noted recurrence of migraine symptomatology with the therapy switch, implying the mechanism of improvement in migraine symptomatology is beyond a direct anticoagulant effect. ${ }^{18}$ In search of a potential non-anticoagulant mechanistic link between warfarin and an apparent improvement in migraine symptomology, we focused on platelets and their ability to secrete 5-HT, as evidence demonstrates that platelet-derived 5-HT may also be involved in migraine pathogenesis. $^{5}$

The precise antiplatelet mechanism of action of warfarin is unclear. However, our data suggest that warfarin may preferentially inhibit platelet 5-HT release over that of aggregation, and that reduced platelet 5-HT secretion falls below the concentration threshold necessary for triggering vasoconstriction of female cerebral vessels, but not of males. Several previous studies have shown that coumarins and other benzopyrones may inhibit aggregation via several mechanisms including inhibiting cyclooxygenase-1 (COX1)/thromboxane $A_{2}$ synthesis, thromboxane $A_{2}$ receptors, and by increasing platelet cAMP levels and nitric oxide production. ${ }^{19-21,27}$ Alternatively, warfarin may inhibit the $\gamma$-carboxylation of platelet-derived growth arrest-specific 6 (Gas6) protein. ${ }^{28}$ Inhibition of this vitamin K-dependent protein is known to dampen aggregation and secretion responses to platelet agonists. ${ }^{29}$ The concentration of warfarin required to inhibit platelet aggregation and 5-HT secretion in our experiments was approximately 10-fold higher than the low micromolar concentrations that are achieved in vivo. ${ }^{30,31}$ However, warfarin is extensively metabolized by the liver via cytochrome P450 enzymes into hydroxylated metabolites, ${ }^{32}$ and hydroxycoumarins have been demonstrated to be starting compounds for the synthesis of derivatives with platelet aggregation inhibitory activity in low micromolar ranges. ${ }^{27,33}$ Hence, in vivo, warfarin may be efficiently metabolized by the liver into metabolites with platelet inhibitory properties. Such metabolites may potentially account for warfarin's occasionally reported, albeit weak, antiplatelet effect in vivo ${ }^{34,35}$ and the need for high warfarin concentrations to produce an antiplatelet effect in vitro (due to less efficient warfarin metabolism by platelets). Interestingly, warfarin had a greater inhibitory effect on platelet 5-HT secretion than aggregation. Pharmacological uncoupling of secretion from platelet aggregation has been previously described for selective inhibitors of protein kinase $\mathrm{C} \alpha$ and inhibitors of actin polymerization, although inhibition of $\alpha$-granule secretion was described. ${ }^{24,36}$ The ability of warfarin or its metabolites to preferentially inhibit platelet secretion over aggregation suggests it may impact components of platelet exocytotic machinery, such as soluble NSF attachment protein receptor 


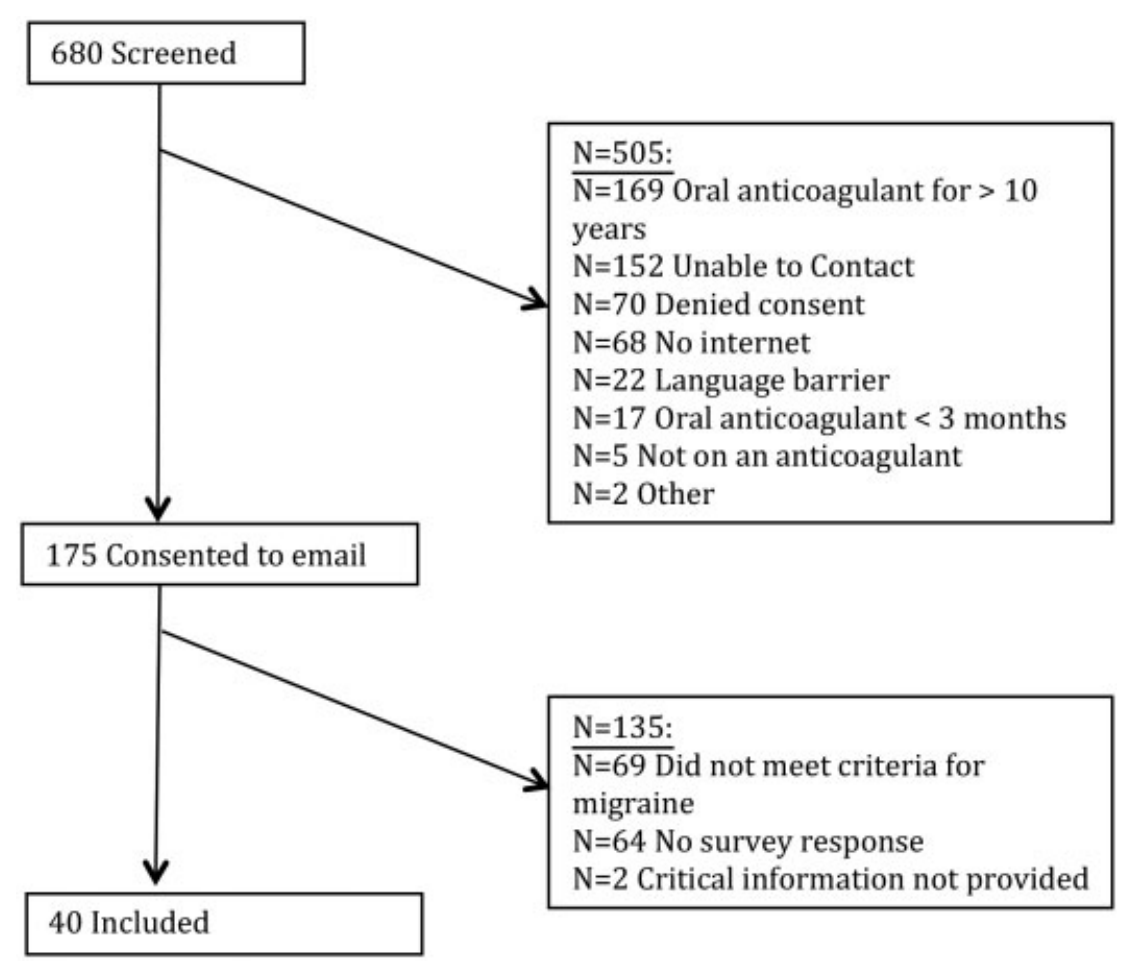

Fig. 3 Patient flow of survey.

(SNARE) proteins or their regulators. Interestingly, warfarin appears to preferentially inhibit $\delta$-granule content secretion ( 5 -HT) over that of the $\alpha$-granules as measured by VEGF. This aspect may contribute to its beneficial effect in improving migraine symptomology in some patients, as 5-HT is a potent vasoconstrictor of human cerebral arteries via the $5-\mathrm{HT}_{1 \mathrm{~B}}$ receptor, ${ }^{37}$ while VEGF is known for its vasodilatory effects. ${ }^{38}$ In addition to warfarin, antiplatelet drugs that also inhibit platelet 5-HT secretion would also be expected to have a similar beneficial antimigraine effect as has been shown in the context of arterial shunt closure with a combination of clopidogrel and ASA. ${ }^{12}$

Several imaging studies have previously reported cerebral hypoperfusion to accompany aura symptoms suggesting involvement of cerebral vasoconstriction, ${ }^{2,3}$ while in our survey those participants reporting an improvement in migraine symptoms were female patients having migraine with aura. Therefore, we investigated the effect of warfarininhibited platelet releasates on vasoconstriction of rat female versus male MCAs to help experimentally explain these apparent sex differences in migraine symptom changes. In response to warfarin-inhibited platelet releasates, which contained reduced 5-HT concentrations, MCAs from male rats demonstrated an eight-fold greater constrictive response than those from female rats. This effect was solely attributed to 5-HT in the platelet releasates as the selective $5-\mathrm{HT}_{2 / 1}$ receptor antagonist ketanserin abolished constriction of male rat MCAs and concentration response experiments confirmed increased sensitivity of male versus female MCAs to the vasoconstrictive effects of 5-HT. This finding is consistent with a previous report demonstrating increased sensitivity of human male versus female cerebral arteries to vasoconstrictor agonists. ${ }^{39}$ Moreover, it has been reported that during attacks of migraine with aura, the initial hypoperfusion accompanying aura is followed by cerebral hyperperfusion, indicative of reactive vasodilation, during the headache phase. ${ }^{3,40}$ The lack of an initial vasoconstrictive response by female cerebral arteries to the reduced concentrations of 5-HT found in warfarin-inhibited platelet releasates may explain the reported improvement in migraine symptomology upon warfarin initiation by female participants with migraine with aura in our study. Among those surveyed, the majority reporting an improvement in migraine symptoms and MIDAS were female patients having migraine with aura and positive smoking history and tended to be of younger age. In contrast, those reporting a worsening in migraine symptomatology were male with half reporting migraines with aura. Notably, among general comments in our survey, three patients reported still having the aura, although had either resolution or an improvement of migraines. The improvement seen with warfarin among those with migraine with aura is interesting in that these patients are also at greater risk of stroke compared with those without aura. ${ }^{17}$

While our survey sought to assess changes in migraine symptomatology that encompassed both an improvement and worsening, others have assessed improvement alone. A Spanish survey of patients assessing improvement in migraine symptomatology (defined as a reduction in frequency by at least $60 \%$ ) with acenocoumarol reported this among 63\% (42/66) of patients. ${ }^{41}$ Those improving were significantly more likely to report vomiting and having severe migraines relative to those reporting no improvement in migraines. It is notable that these authors correlate the 
Table 1 Demographics

\begin{tabular}{|c|c|c|}
\hline Factor & $\begin{array}{l}\text { Change in migraine } \\
\text { symptoms, } N=11\end{array}$ & $\begin{array}{l}\text { No change in migraine } \\
\text { symptoms, } N=29\end{array}$ \\
\hline \multicolumn{3}{|l|}{ Age } \\
\hline$<36 y$ & 2 & 2 \\
\hline $36-45$ y & 1 & 4 \\
\hline $46-55$ y & 5 & 6 \\
\hline $56-65$ y & 1 & 11 \\
\hline$>65 y$ & 2 & 6 \\
\hline \multicolumn{3}{|l|}{ Gender } \\
\hline Female & 4 & 15 \\
\hline Male & 7 & 14 \\
\hline \multicolumn{3}{|l|}{ Type of migraine } \\
\hline With aura & 8 & 6 \\
\hline Without aura & 1 & 9 \\
\hline Diagnosis only & 2 & 14 \\
\hline Years since onset of migraine (median, IQR) & $21(10-29)$ & $32(21-41)$ \\
\hline Duration of anticoagulant (median, IQR) & $3(1-6)$ & $5(1-8)$ \\
\hline \multicolumn{3}{|l|}{ Anticoagulant } \\
\hline Warfarin & 11 & 27 \\
\hline Dabigatran & 0 & 1 \\
\hline Rivaroxaban & 0 & 1 \\
\hline \multicolumn{3}{|l|}{ Indication for anticoagulant ${ }^{\mathrm{a}}$} \\
\hline Mechanical valve & 10 & 21 \\
\hline Atrial fibrillation & 1 & 7 \\
\hline Venous thromboembolism & 0 & 1 \\
\hline Stroke & 0 & 2 \\
\hline Other & $1^{\mathrm{b}}$ & $2^{c}$ \\
\hline \multicolumn{3}{|l|}{ Medical history } \\
\hline Congenital heart disease & 7 & 16 \\
\hline Hypertension & 4 & 14 \\
\hline Hyperlipidemia & 1 & 8 \\
\hline Diabetes & 1 & 7 \\
\hline Heart failure & 2 & 7 \\
\hline Stroke/TIA & 1 & 5 \\
\hline Myocardial infarction & 1 & 1 \\
\hline Current smoker & 0 & 2 \\
\hline Past smoker & 4 & 15 \\
\hline Chronic daily ASA use with anticoagulant & 4 & 11 \\
\hline
\end{tabular}

Abbreviations: ASA, acetyl salicylic acid; IQR, interquartile range; TIA, transient ischemic attack.

${ }^{\text {a }}$ Not mutually exclusive.

${ }^{\mathrm{b}}$ One portal vein thrombosis.

'One cerebral venous sinus thrombosis and one portal vein thrombosis.

clinical improvement in migraines with the severity of attacks. In our study, the resultant change in the MIDAS was also clinically significant in that the change in scores would result in a change in the grade of disability. The magnitude of impact of clinical improvement with VKA administration is further substantiated by case reports of patients wanting to stay on warfarin, despite having no further indication. ${ }^{17,42}$

The general patient characteristics (age, gender, and migraine with aura) among those showing improvement in 
Table 2 Characterization based on change in MIDAS

\begin{tabular}{|c|c|c|c|}
\hline Factor & $\begin{array}{l}\Delta-\text { MIDAS } \\
N=5\end{array}$ & $\begin{array}{l}\Delta+\text { MIDAS } \\
N=4\end{array}$ & $\begin{array}{l}\text { No } \Delta \text { MIDAS } \\
N=2\end{array}$ \\
\hline Median change in MIDAS (range) & $-19(-24$ to -8$)$ & $24(14-40)$ & - \\
\hline \multicolumn{4}{|l|}{ Gender } \\
\hline Female & 4 & 0 & 0 \\
\hline Male & 1 & 4 & 2 \\
\hline \multicolumn{4}{|l|}{ Type of migraine } \\
\hline With aura & 4 & 2 & 2 \\
\hline Without aura & 0 & 1 & 0 \\
\hline Diagnosis only & 1 & 1 & 0 \\
\hline Smoking status-past & 3 & 1 & 0 \\
\hline \multicolumn{4}{|l|}{ Indication for anticoagulant } \\
\hline Mechanical valve & 4 & 4 & 2 \\
\hline Atrial fibrillation & 1 & 0 & 0 \\
\hline Venous thromboembolism & 0 & 0 & 0 \\
\hline Stroke & 0 & 0 & 0 \\
\hline \multicolumn{4}{|l|}{ Medical history } \\
\hline Congenital heart disease & 3 & 3 & 1 \\
\hline Hypertension & 3 & 0 & 1 \\
\hline Hyperlipidemia & 1 & 0 & 0 \\
\hline Diabetes & 0 & 0 & 1 \\
\hline Heart failure & 0 & 2 & 0 \\
\hline Stroke & 0 & 1 & 0 \\
\hline Myocardial infarction & 0 & 0 & 1 \\
\hline
\end{tabular}

Abbreviation: MIDAS, Migraine Disability Assessment Score.

Note: A reduction $(\Delta-)$ in MIDAS demonstrates an improvement, whereas an increase $(\Delta+)$ demonstrates worsening of migraine symptoms.

migraine symptomatology in our study are consistent with other literature reports. Of the 16 case reports of an improvement in migraine symptomatology with VKA therapy, 13 were less than 50 years of age (range of 24-46 years) ${ }^{15,17,26,42,43}$ with the remaining 3 being 55,68 , and 71 years of age. ${ }^{13,14,18}$ Only one of these cases was male. ${ }^{15}$ Among the eight reports that provide a description of the migraine, seven cases report improvement and were identified as having migraine with aura. ${ }^{13-15,17,18,42,43}$ While not all cases describe specific symptomatology changes, a few cases describe the ongoing presence of an aura with resolution/improvement in migraine severity/ frequency, ${ }^{15,17}$ as reported in our survey.

As younger age was also associated with improvement in migraine symptomology among these participants, sex hormones may play a major role in this warfarin-mediated improvement. Estrogen, well known for its vasodilatory and vascular protective function, ${ }^{44,45}$ may counteract the constrictive effects of 5-HT in younger female vessels. Hence, a warfarin-induced reduction in platelet 5-HT secretion coupled with a reduced response to low concentrations of 5-HT by estrogen-protected female vessels may be responsible for the improvement in symptomology. Reduced platelet 5-HT secretion by warfarin may also improve migraine symptomology beyond a vascular mechanism, as 5-HT also plays important roles in nociception and neurogenic/neurovascular inflammation. ${ }^{46,47}$

Our study has a few limitations. Specific to the patient survey, we are subject to both selection bias in that patients having a change in migraine symptoms with warfarin initiation may have been more likely to respond to our survey. Recall bias may have also occurred, as we did recruit patients who had started an oral anticoagulant as long as 10 years ago. However, selection and recall bias would be expected to affect migraine patients with and without aura as well as females and males similarly. In our study, it is worth noting that patients reporting a change in migraine symptoms were predominantly those with an aura component, and generally female and male patients reported opposite responses (improvement in migraine symptoms vs. worsening), suggesting survey bias did not influence our results. Further support for a causal relationship between improvement in migraine symptomatology and VKA use are the general consistencies in the timing of symptom change as well as cases of re-challenge that support an effect. While our study had patients focus on changes within 3 months of anticoagulant change, others have reported more specific timing of symptom change. While the timing of change is dependent on an individual patient's baseline frequency of migraine occurrence, one case reported 
an improvement in 2 to 3 days, ${ }^{18}$ with others reported changes within a month. ${ }^{13,14,17,42}$ Similarly, for those discontinuing a VKA, symptom recurrence is reported within 10 days to a month. ${ }^{13,15,18,42}$ In terms of re-challenge with the VKA to discern consistency of change, three case reports have demonstrated this ${ }^{14,15,18}$ and one double-blind trial in a patient administering either placebo for 8 weeks (2-3 migraines per week) or warfarin targeted to an INR of 2.0 to 3.0 (migraine resolution). ${ }^{13}$ Following the phase wherein the INR was targeted to 2.0 to 3.0 (administration of warfarin $6 \mathrm{mg}$ daily), a warfarin dose reduction to $3 \mathrm{mg}$ daily (yielding INRs between 1 and 1.2) maintained the resolution of migraines.

Another limitation of our study is that MIDAS is a clinical assessment tool to detect differences in level of disability and not necessarily the symptomatology of a migraine. Two patients reported a change in their migraine symptomatology without a resultant change in their MIDAS, and we are not able to discern (based on survey design) if the symptom change was an improvement or worsening. Literature indicates that clinical improvement in migraines with VKA use is correlated with the severity of the attacks (survey) ${ }^{41,42}$ perhaps, suggesting the symptom change occurring in these patients may have been less severe migraine symptomatology. Similarly, there are several limitations to our mechanistic ex vivo experiments including utilizing healthy donor platelets and rat MCAs, while also strictly focusing on platelet and vascular aspects of migraine pathophysiology. Nonetheless, our mechanistic experiments may be a starting point for animal model studies that may lead to development of novel VKA-dependent therapies for migraine with aura.

Finally, as patients with migraine with aura are at greater risk of ischemic stroke and venous thromboembolism than those without aura, ${ }^{17,48,49}$ warfarin may be the anticoagulant of choice in these female patients providing both migraine relief and thrombotic protection.

\section{Funding}

This work was funded by grants from the Canadian Institutes of Health Research MOP-130289 (P.J.). We thank S. Simpson for assistance with survey.

\section{Conflict of Interest}

T.J.B. has received speaker honoraria from Bayer and unrestricted research grants from Pfizer and Leo Pharma, none of which relates to the content of this manuscript. The remaining authors have no conflicts to declare.

\section{References}

1 Wolff HG. Headache and Other Pain. 2nd ed. New York, NY: Oxford University Press; 1963

2 Andersen AR, Friberg L, Olsen TS, Olesen J. Delayed hyperemia following hypoperfusion in classic migraine. Single photon emission computed tomographic demonstration. Arch Neurol 1988;45 (02):154-159

3 Olesen J, Friberg L, Olsen TS, et al. Timing and topography of cerebral blood flow, aura, and headache during migraine attacks. Ann Neurol 1990;28(06):791-798

4 Ferrari MD. Migraine. Lancet 1998;351(9108):1043-1051
5 Danese E, Montagnana M, Lippi G. Platelets and migraine. Thromb Res 2014;134(01):17-22

6 Buttinelli C, Lazzaro MP, Lenzi GL, Paolucci S, Prencipe M. Correlation between migraine and circulating platelet aggregates. Cephalalgia 1985;5(02, Suppl 2):87-88

7 Lechner H, Ott E, Fazekas F, Pilger E. Evidence of enhanced platelet aggregation and platelet sensitivity in migraine patients. Cephalalgia 1985;5(02, Suppl 2):89-91

8 Tietjen GE, Al-Qasmi MM, Athanas K, Utley C, Herial NA. Altered hemostasis in migraineurs studied with a dynamic flow system. Thromb Res 2007;119(02):217-222

9 Mück-Seler D, Deanović Z, Dupelj M. Platelet serotonin (5-HT) and 5-HT releasing factor in plasma of migrainous patients. Headache 1979;19(01):14-17

10 Benemei S, Cortese F, Labastida-Ramírez A, et al; School of Advanced Studies of the European Headache Federation (EHFSAS). Triptans and CGRP blockade: impact on the cranial vasculature. J Headache Pain 2017;18(01):103

11 Savi LT, Condello V, Bert F, Pinessi L. Prophylaxis of migraine with aura: a place for acetylsalicylic acid. J Headache Pain 2013;14 (Suppl 1):P195

12 Wilmshurst PT, Nightingale S, Walsh KP, Morrison WL. Clopidogrel reduces migraine with aura after transcatheter closure of persistent foramen ovale and atrial septal defects. Heart 2005;91 (09):1173-1175

13 Suresh CG, Neal D, Coupe MO. Warfarin treatment and migraine. Postgrad Med J 1994;70(819):37-38

14 van Puijenbroek EP, Egberts AC, Trooster JF, Zomerdijk J. Reduction of migrainous headaches during the use of acenocoumarol. Headache 1996;36(01):48-48

15 Fragoso YD. Reduction of migraine attacks during the use of warfarin. Headache 1997;37(10):667-668

16 Souza JA, Moreira Filho PF, Jevoux Cda C, Martins GF, Pitombo AB. Remission of refractory chronic cluster headache after warfarin administrations: case report. Arq Neuropsiquiatr 2004;62(04): 1090-1091

17 Russo A, Santi S, Gueraldi D, De Paola M,Zani F, Pini LA. An unusual case report on the possible role of Warfarin in migraine prophylaxis. Springerplus 2013;2(01):48

18 Nilsson BG, Bungard TJ. A case of migraine with aura resolving on warfarin but not on apixaban. Headache 2017;57(10):1614-1617

19 Teng CM, Li HL, Wu TS, Huang SC, Huang TF. Antiplatelet actions of some coumarin compounds isolated from plant sources. Thromb Res 1992;66(05):549-557

20 Leoncini G, Signorello MG, Bruzzese D, Di Braccio M, Grossi GC, Roma G. Mechanisms involved in the antiplatelet activity of 8methyl-4-(1-piperazinyl)-7-(3-pyridinylmethoxy)-2H-1-benzopyran-2-one (RC414). Biochem Pharmacol 2004;67(05):911-918

21 Macáková K, Řeháková Z, Mladěnka $P$, et al. In vitro platelet antiaggregatory properties of 4-methylcoumarins. Biochimie 2012;94(12):2681-2686

22 Schürks M, Rist PM, Bigal ME, Buring JE, Lipton RB, Kurth T. Migraine and cardiovascular disease: systematic review and meta-analysis. BMJ 2009;339:b3914

23 Spector JT, Kahn SR, Jones MR, Jayakumar M, Dalal D, Nazarian S. Migraine headache and ischemic stroke risk: an updated metaanalysis. Am J Med 2010;123(07):612-624

24 Moncada de la Rosa C, Radziwon-Balicka A, El-Sikhry H, et al. Pharmacologic protein kinase $\mathrm{C} \alpha$ inhibition uncouples human platelet-stimulated angiogenesis from collagen-induced aggregation. J Pharmacol Exp Ther 2013;345(01):15-24

25 Stewart WF, Lipton RB, Whyte J, et al. An international study to assess reliability of the Migraine Disability Assessment (MIDAS) score. Neurology 1999;53(05):988-994

26 Wammes-van der Heijden EA, Tijssen CC, van't Hoff AR, Egberts AC. A thromboembolic predisposition and the effect of anticoagulants on migraine. Headache 2004;44(05):399-402 
27 Di Braccio M, Grossi G, Roma G, Grazia Signorello M, Leoncini G. Synthesis and in vitro inhibitory activity on human platelet aggregation of novel properly substituted 4-(1-piperazinyl)coumarins. Eur J Med Chem 2004;39(05):397-409

28 Ishimoto Y, Nakano T. Release of a product of growth arrest-specific gene 6 from rat platelets. FEBS Lett 2000;466(01):197-199

29 Angelillo-Scherrer A, de Frutos P, Aparicio C, et al. Deficiency or inhibition of Gas6 causes platelet dysfunction and protects mice against thrombosis. Nat Med 2001;7(02):215-221

30 Chan E, McLachlan AJ, Pegg M, MacKay AD, Cole RB, Rowland M. Disposition of warfarin enantiomers and metabolites in patients during multiple dosing with rac-warfarin. Br J Clin Pharmacol 1994;37(06):563-569

31 Tiseo PJ, Foley K, Friedhoff LT. The effect of multiple doses of donepezil $\mathrm{HCl}$ on the pharmacokinetic and pharmacodynamic profile of warfarin. Br J Clin Pharmacol 1998;46(01, Suppl 1):45-50

32 Shaik AN, Grater R, Lulla M, et al. Comparison of enzyme kinetics of warfarin analyzed by LC-MS/MS QTrap and differential mobility spectrometry. J Chromatogr B Analyt Technol Biomed Life Sci 2016;1008:164-173

33 Chen YL, Wang TC, Liang SC, Teng CM, Tzeng CC. Synthesis and evaluation of coumarin alpha-methylene-gamma-butyrolactones: a new class of platelet aggregation inhibitors. Chem Pharm Bull (Tokyo) 1996;44(08):1591-1595

34 Kovacs IB, Murphy M, Barin E, Rees GM. Effect of warfarin on arterial thrombogenesis: problems of monitoring. Am J Hematol 1993;42(03):322-327

35 Newland H, Nordöy A. Effect of large doses of warfarin sodium on haemostasis and on ADP-induced platelet aggregation in vivo in the rat. A survey of experimental thrombosis and coumarin anticoagulant therapy. Cardiovasc Res 1967;1(04):362-370

36 Woronowicz K, Dilks JR, Rozenvayn N, et al. The platelet actin cytoskeleton associates with SNAREs and participates in alphagranule secretion. Biochemistry 2010;49(21):4533-4542

37 Nilsson T, Longmore J, Shaw D, Olesen IJ, Edvinsson L. Contractile 5-HT1B receptors in human cerebral arteries: pharmacological characterization and localization with immunocytochemistry. $\mathrm{Br}$ J Pharmacol 1999;128(06):1133-1140

38 Ku DD, Zaleski JK, Liu S, Brock TA. Vascular endothelial growth factor induces EDRF-dependent relaxation in coronary arteries. Am J Physiol 1993;265(2, Pt 2):H586-H592

39 Ahnstedt H, Cao L, Krause DN, et al. Male-female differences in upregulation of vasoconstrictor responses in human cerebral arteries. PLoS One 2013;8(04):e62698

40 Hansen JM, Schankin CJ. Cerebral hemodynamics in the different phases of migraine and cluster headache. J Cerebral Blood Flow Metabol 2019;39(04):595-609

41 Morales-Asín F, Iñiguez C, Cornudella R, Mauri JA, Espada F, Mostacero EE. Patients with acenocoumarol treatment and migraine. Headache 2000;40(01):45-47

42 Maggioni F, Bruno M, Mainardi F, Lisotto C, Zanchin G. Migraine responsive to warfarin: an update on anticoagulant possible role in migraine prophylaxis. Neurol Sci 2012;33(06):1447-1449

43 Cuadrado MJ, Khamashta MA, Hughes GRV. Migraine and stroke in young women. QJM 2000;93(05):317-318

44 White RE. Estrogen and vascular function. Vascul Pharmacol 2002;38(02):73-80

45 Iorga A, Cunningham CM, Moazeni S, Ruffenach G, Umar S, Eghbali $M$. The protective role of estrogen and estrogen receptors in cardiovascular disease and the controversial use of estrogen therapy. Biol Sex Differ 2017;8(01):33

46 Viguier F, Michot B, Hamon M, Bourgoin S. Multiple roles of serotonin in pain control mechanisms-implications of $5-\mathrm{HT}_{7}$ and other 5-HT receptor types. Eur J Pharmacol 2013;716(1-3):8-16

47 Segelcke D, Messlinger K. Putative role of $5-\mathrm{HT}_{2 \mathrm{~B}}$ receptors in migraine pathophysiology. Cephalalgia 2017;37(04):365-371

48 Peng KP, Chen YT, Fuh JL, Tang CH, Wang SJ. Migraine and incidence of ischemic stroke: a nationwide population-based study. Cephalalgia 2017;37(04):327-335

49 Peng KP, Chen YT, Fuh JL, Tang CH, Wang SJ. Association between migraine and risk of venous thromboembolism: a nationwide cohort study. Headache 2016;56(08):1290-1299 